\title{
Neuroelectric Current Localization from Combined EEG/MEG Data
}

\author{
Francesca Pitolli \\ Dept. SBAI, University of Roma "La Sapienza", \\ Via A. Scarpa 1, 00161 Roma, Italy \\ pitolli@dmmm.uniroma1.it \\ http://www.dmmm.uniroma1.it/ pitolli
}

\begin{abstract}
EEG/MEG devices record external signals which are generated by the neuronal electric activity of the brain. The localization of the neuronal sources requires the solution of the neuroelectromagnetic inverse problem which is highly ill-posed and ill-conditioned.

We provide an iterative thresholding algorithm for recovering neuroeletric current densities within the brain through combined EEG/MEG data. We use a joint sparsity constraint to promote solutions localized in small brain area, assuming that the vector components of the current densities possess the same sparse spatial pattern. At each iteration step, the EEG/MEG forward problem is numerically solved by a Galerkin boundary element method. Some numerical experiments on the localization of current dipole sources are also given.

The numerical results show that joint sparsity constraints outperform classical regularization methods based on quadratic constraints.
\end{abstract}

Keywords: Source reconstruction, Sparse representation, Thresholded iteration, Galerkin boundary element method

\section{Introduction}

Functional neuroimaging aims at understanding human brain functionality through the localization of the active regions of the brain, for instance, during specific tasks or even during rest. Commonly used neuroimaging techniques, such as single-photon emission computed tomography (SPECT) and positron-emission tomography (PET), make use of invasive devices which expose the subject to $\mathrm{x}$ rays and radioactive tracers, respectively. Moreover, these techniques, being related to the chemical reactions that take place inside the brain, are not able to follow rapid changes occurring in neuronal activity. For this reason, in neuroscience studies there is a great effort in developing imaging techniques with higher temporal resolution.

In recent years, magnetoencephalography (MEG) has gained an important role in the field of neuroscience research since it is completely noninvasive and

\footnotetext{
* Preprint 2011: submitted to the Proceedings of Seventh International Conference on Curves and Surfaces, Avignon, France, June 24-30, 2010
} 
has a high temporal resolution. MEG aims at identifying the active area of the brain by localizing inner electric current sources through the measurements of the tiny magnetic field generated externally of the head by neuronal electric activity (see the review papers $[10,20]$ for details). MEG is not a proper imaging technique since to recover the current distribution underlying the magnetic data an inverse problem has to be solved. It is known that the solution of the magnetic inverse problem is nonunique, since there exists silent sources which do not produce any external magnetic field [22]. Moreover, the magnetometers are just a few hundreds, while in the localization a high spatial resolution is required, and the measurements are affected by high noise. Thus, the MEG localization problem results in an highly ill-posed and ill-conditioned inverse problem and it is mandatory to use suitable regularization methods for its numerical solution $[4,13,22]$.

Since neuronal sources are localized in small regions, the electric current flowing in the brain can be assumed to have a sparse spatial representation, i.e. it can be represented as a sum of weighted basic currents, encoded by the position and the spatial scale, in which just few terms are relevant. To promote sparse solutions, regularization methods based on sparsity constraints look promising $[8,11]$. In particular, since the electric current density is a vectorvalued function, it makes sense to assume that all its three components have the same sparse spatial structure and to promote the same sparsity pattern on all of them. To this end, joint sparsity constraints to reconstruct multichannel signals were considered in $[15,16]$ where an iterative thresholding algorithm to recover sparse vector-valued functions is also given. Following the same strategy, in [14] an iterative thresholding algorithm especially designed to solve the MEG inverse problem was proposed. Several numerical tests $[5,25]$ have shown that this algorithm outperforms classical regularization methods based on quadratic constraints $[4,13]$ in localizing current dipole sources, which are usually used to model neuroelectric currents.

In [14] just the magnetic inverse problem was considered. However, the neuronal activity generating the external magnetic field is responsible for the electric potential differences on the scalp, too. Thus, a strategy to gain some further information on neuroelectric current distributions inside the brain consists in measuring also the electric potential differences by carrying out standard (noninvasive) electroencephalografic (EEG) records during MEG measurements. Moreover, the electric and magnetic field are mutually orthogonal so that combined EEG/MEG measurements allows to detect sources which would be silent w.r.t. EEG or MEG alone. For instance, neuroelectric sources radially oriented w.r.t. the skull surface are magnetically silent but can be detected by electric measurements. On the other hand, current loops are electrically silent but produce an external magnetic field. Thus, EEG and MEG data are complementary and best results in recovering brain functionality are obtained by integrating information from both techniques [2].

In this paper we present a numerical method to solve the neuroelectromagnetic inverse problem, i.e. the reconstruction of a neuroelectric current distri- 
bution from combined EEG/MEG data, using a joint sparsity constraints as a regularization technique. The neuroelectromagnetic field can be described by the quasistatic Maxwell's equations which give rise to a boundary integral equation for the electric potential and to the Ampère-Laplace law for the magnetic field $[18,19]$. The integral operator equations describing the EEG/MEG forward problem will be carried out in Sec. 2. In Sec. 3 we will set the EEG/MEG inverse problem with a joint sparsity constraint and we will provide an iterative thresholding algorithm for its numerical solution that generalizes to the combined EEG/MEG inverse problem the algorithm developed in [14].

At each iteration step, we need to solve the EEG/MEG forward problem, i.e. the evaluation of the electric potential difference and the magnetic field once the neuroelectric current distribution in the brain is given. In neuroscience literature, the EEG forward problem is usually solved by a collocation boundary element method (BEM) in which the electric current density is modeled as a sum of current dipoles with given positions $[2,17]$. Here, we will follow a different approach: we will present a Galerkin boundary element method where we do not limit ourselves to a specific form of the current density. In this way we expect to be able to resolve not only dipole sources, but also electric current densities with different pattern, for instance, current loop that in recent studies are assumed to connect different brain regions. Details on the Galerkin BEM for the solution of the EEG/MEG forward problem will be given in Sec. 4. Finally, in Sec. 5 some numerical results on a simple test problem will be displayed and some conclusion and perspectives will be given.

\section{The EEG/MEG Forward Problem}

The neuroelectromagnetic field, i.e. the electromagnetic field generated by neuronal electric activity, can be describe by the Maxwell's equations. Actually, focusing on biological conductors some simplifying assumptions can be taken into account. Firstly, the permeability of the tissues in the head (scalp, skull, cerebrospinal fluid and brain) is the same as the permeability in the free space, say $\mu_{0}$. Moreover, there are no electric charges in the conducting medium. Finally, due to the low frequencies of bioelectric phenomena, we may neglect the time derivatives of the electric and the magnetic fields.

Thus, the quasistatic Maxwell's equations in a tissue with electric permittivity $\varepsilon_{0}$ and magnetic permeability $\mu_{0}$ read

$$
\begin{array}{cc}
\nabla \cdot \mathbf{E}=0, & \nabla \cdot \mathbf{B}=0, \\
\nabla \times \mathbf{E}=0, & \nabla \times \mathbf{B}=\mu_{0} \mathbf{J},
\end{array}
$$

where $\mathbf{E}$ and $\mathbf{B}$ are the electric and magnetic field, respectively, and $\mathbf{J}$ is the electric current density inside the tissue. As usual, we may express the irrotational electric field with a scalar potential, i.e.

$$
\mathbf{E}=-\nabla V .
$$


The EEG/MEG forward problem is to evaluate the neuroelectric potential $V$ and the neuromagnetic field $\mathbf{B}$ once the electric current sources are given. It results in solving (2)-(3) once $\mathbf{J}$ is given.

The current density $\mathbf{J}$ flowing in the brain has two components. The primary current $\mathbf{J}^{p}$, generated by neuronal activity, flows inside or in the vicinity of the neurons. The volume current $\mathbf{J}^{v}(\mathbf{r})=\sigma(\mathbf{r}) \mathbf{E}(\mathbf{r})$ flows passively everywhere in the medium that is assumed to have macroscopic conductivity $\sigma(\mathbf{r})$. Thus,

$$
\mathbf{J}(\mathbf{r})=\mathbf{J}^{p}(\mathbf{r})+\sigma(\mathbf{r}) \mathbf{E}(\mathbf{r})=\mathbf{J}^{p}(\mathbf{r})-\sigma(\mathbf{r}) \nabla V(\mathbf{r}) .
$$

Following [18],[19] we model the head as a conductor consisting of homogeneous nested regions, each one having constant conductivity. Let $G_{i}, i=$ $0, \ldots, m$, be the regions and $\sigma_{i}, i=0, \ldots, m$, be the constant conductivity inside $G_{i}$. Let us denote by $S_{i}, i=0, \ldots, m$, the interfaces between $G_{i}$ and $G_{i+1}$ with $\mathbf{n}_{i}(\mathbf{r})$ being the unit vector perpendicular to $\partial G_{i}$ in $\mathbf{r}$ and pointing at $G_{i+1}$. We assume the regions are nested with $G_{0}$ being the innermost region, i.e.

$$
G_{0} \subset G_{1} \subset \cdots \subset G_{m}
$$

and the interfaces $S_{i}, i=0, \ldots, m$, are not intersecting. The primary current $\mathbf{J}^{p}$ flows just inside $G_{0}$.

From the Maxwell's equations (see [20] for details) it follows that the electric current density (4) and the external magnetic field are related by the AmpèreLaplace law that reads

$$
\mathbf{B}(\mathbf{r})=\mathbf{B}_{0}(\mathbf{r})-\frac{\mu_{0}}{4 \pi} \sum_{i=1}^{m} \sigma_{i} \int_{G_{i} \backslash G_{i-1}} \nabla V\left(\mathbf{r}^{\prime}\right) \times \frac{\mathbf{r}-\mathbf{r}^{\prime}}{\left|\mathbf{r}-\mathbf{r}^{\prime}\right|^{3}} d \mathbf{r}^{\prime},
$$

where

$$
\mathbf{B}_{0}(\mathbf{r})=\frac{\mu_{0}}{4 \pi} \int_{G_{0}} \frac{\mathbf{J}^{p}\left(\mathbf{r}^{\prime}\right) \times\left(\mathbf{r}-\mathbf{r}^{\prime}\right)}{\left|\mathbf{r}-\mathbf{r}^{\prime}\right|^{3}} d \mathbf{r}^{\prime}
$$

is the magnetic field produced by $\mathbf{J}^{p}$ in an infinite homogeneous medium. The volume integrals in (5) can be transformed into surface integrals on the interfaces [19] obtaining

$$
\mathbf{B}(\mathbf{r})=\mathbf{B}_{0}(\mathbf{r})-\frac{\mu_{0}}{4 \pi} \sum_{i=0}^{m}\left(\sigma_{i+1}-\sigma_{i}\right) \int_{S_{i}} V\left(\mathbf{r}^{\prime}\right) \frac{\mathbf{r}-\mathbf{r}^{\prime}}{\left|\mathbf{r}-\mathbf{r}^{\prime}\right|^{3}} \times \mathbf{n}_{i}\left(\mathbf{r}^{\prime}\right) d S_{i}\left(\mathbf{r}^{\prime}\right),
$$

(in the sum we may assume $\sigma_{m+1}=0$ ).

It can be shown [20] that the electric potential $V$ satisfies a surface integral equation which involves $V$ at the interfaces only:

$$
\begin{array}{r}
\frac{\left(\sigma_{l+1}+\sigma_{l}\right)}{2} V(\mathbf{r})=\sigma_{0} V_{0}(\mathbf{r})+\frac{1}{4 \pi} \sum_{i=1}^{m}\left(\sigma_{i+1}-\sigma_{i}\right) \int_{S_{i}} V\left(\mathbf{r}^{\prime}\right) \frac{\mathbf{r}-\mathbf{r}^{\prime}}{\left|\mathbf{r}-\mathbf{r}^{\prime}\right|^{3}} \cdot \mathbf{n}_{i}\left(\mathbf{r}^{\prime}\right) d S_{i}\left(\mathbf{r}^{\prime}\right) \\
\mathbf{r} \in S_{l}, l=0, \ldots, m
\end{array}
$$


where

$$
V_{0}(\mathbf{r})=\frac{1}{4 \pi \sigma_{0}} \int_{G_{0}} \frac{\mathbf{J}^{p}\left(\mathbf{r}^{\prime}\right) \cdot\left(\mathbf{r}-\mathbf{r}^{\prime}\right)}{\left|\mathbf{r}-\mathbf{r}^{\prime}\right|^{3}} d \mathbf{r}^{\prime}
$$

is the electric potential produced by $\mathbf{J}^{p}$ in an infinite homogeneous medium [18].

Equations (6)-(9) can be solved analytically for a few simple current distributions and conductor geometry. For instance, an analytical solution of the forward problem can be obtained when the sources are modeled as current dipoles in a homogeneous conducting sphere [26]. More realistic head models require numerical methods to solve the integral equation (8) and to evaluate (7). This will be addressed in Sec. 4.

\section{The EEG/MEG Inverse Problem}

In order to gain information on brain functionality we need to reconstruct the primary current density $\mathbf{J}^{p}$ from the measured data. This results in an inverse problem, known as neuroelectromagnetic inverse problem, which is to estimate the brain current sources underlying the measurements of the scalp electric potential and the external magnetic field [10],[20].

Let $\mathbf{q}_{i}, i=1, \ldots, M$, and $\mathbf{p}_{i}, i=1, \ldots, N$, be the magnetometer and electrode sites, respectively. The sites $\mathbf{q}_{i}, i=1, \ldots, M$, belong to a surface $\Sigma$ with $\operatorname{dist}\left(\Sigma, G_{m}\right)>0$, and each magnetometer measures the magnetic field $B_{i}$, $i=1, \ldots, M$, along the direction $\mathbf{e}\left(\mathbf{q}_{i}\right)$ (usually the normal to the magnetometer coil). The sites $\mathbf{p}_{i}, i=1, \ldots, N$, belong to the surface $G_{m}$ (the scalp), and each electrode measures the potential difference $V_{i}, i=1, \ldots, N$, w.r.t. a reference electrode.

Now, let $\mathcal{B}_{\mathbf{e}}\left(\mathbf{r}, \mathbf{J}^{p}\right):=\mathbf{B}(\mathbf{r}) \cdot \mathbf{e}(\mathbf{r})$ and $\mathcal{V}\left(\mathbf{r}, \mathbf{J}^{p}\right):=V(\mathbf{r})$ be the integral operators representing the solution of the forward problem (7)-(8). We note that both $\mathcal{B}_{\mathbf{e}}\left(\mathbf{r}, \mathbf{J}^{p}\right)$ and $\mathcal{V}\left(\mathbf{r}, \mathbf{J}^{p}\right)$ are linearly related to $\mathbf{J}^{p}$. Thus, the neuroelectromagnetic inverse problem is to minimize the discrepancy

$$
\Delta\left(\mathbf{J}^{p}\right)=\sum_{i=1}^{M}\left(B_{i}-\mathcal{B}_{\mathbf{e}}\left(\mathbf{q}_{i}, \mathbf{J}^{p}\right)\right)^{2}+\sum_{i=1}^{N}\left(V_{i}-\mathcal{V}\left(\mathbf{p}_{i}, \mathbf{J}^{p}\right)\right)^{2}
$$

w.r.t. to the primary current distribution $\mathbf{J}^{p}$.

We recall that a current distribution inside a conductor cannot be retrieved uniquely from knowledge of the electromagnetic field outside (see, for instance, $[20,22])$. There are primary current distributions that are either magnetically, or electrically silent, or both, i.e. there may exist neuronal currents that do not produce any external magnetic field or electric potential differences on the head. Thus, we must add some further constraints in order to confine ourselves to find a solution among a limited class of source configurations [4],[13].

The regularization methods based on quadratic constraints lead to over smoothed source estimations $[8,12]$. In particular, minimum $\ell_{2}$-norm estimates, i.e. minimizers of the functional

$$
\Delta\left(\mathbf{J}^{p}\right)+\alpha\left\|\mathbf{J}^{p}\right\|_{2}^{2}, \quad \alpha>0,
$$


are not appropriate for the localization of epileptic foci, which are known to be confined in small brain regions. This allows us to assume that the primary current has a sparse representation w.r.t. a suitable basis of compactly supported functions $\left(\psi_{\lambda}\right)_{\lambda \in \Lambda}$, i.e.

$$
\mathbf{J}^{p}=\left(J^{1}, J^{2}, J^{3}\right) \in L_{2}\left(G_{0} ; \mathbb{R}^{3}\right), \quad J^{\ell}=\sum_{\lambda \in \Lambda} j_{\lambda}^{\ell} \psi_{\lambda}, \quad \ell=1,2,3,
$$

where only few coefficients $\left(j_{\lambda}^{\ell}\right)$ for each component are non-vanishing [11].

We note that multiscale basis are successfully used in medical signal processing and image analysis since scale adaptivity allows to detect spikes and discontinuities appearing in biological signals or inhomogeneous structures characterizing biological tissues [1]. Thus, we may assume $\left(\psi_{\lambda}\right)_{\lambda \in \Lambda}$ be a multiscale basis, i.e. a wavelet basis or a frame [23].

Since all the components $J^{\ell}, \ell=1,2,3$, are related to the same neurophysiological phenomenon, it makes sense to assume the subset of non-vanishing coefficients being the same for all the three components. This is equivalent to require that $\mathbf{J}^{p}$ has a sparse representation w.r.t. the joint $\ell_{q}$-norm, defined as

$$
\left\|\left(\mathbf{j}_{\lambda}\right)_{\lambda \in \Lambda}\right\|_{q}:=\left(\sum_{\lambda \in \Lambda}\left(\left\|\mathbf{j}_{\lambda}\right\|_{\mathbb{R}^{3}}\right)^{q}\right)^{1 / q}, \quad q \geq 1,
$$

where $\mathbf{j}_{\lambda}=\left(j_{\lambda}^{1}, j_{\lambda}^{2}, j_{\lambda}^{3}\right)^{T}[15]$.

Let $\mathbf{j}=\left(\mathbf{j}_{\lambda}\right)_{\lambda \in \Lambda}$. Following [15], it can be shown that the solution of the EEG/MEG inverse problem with a joint sparsity constraint is the minimizer of the functional

$$
\mathcal{J}_{\theta, \rho, \omega}^{(q)}(\mathbf{j}, v):=\Delta(\mathbf{j})+\left(\sum_{\lambda \in \Lambda} v_{\lambda}\left\|\mathbf{j}_{\lambda}\right\|_{q}+\sum_{\lambda \in \Lambda} \omega_{\lambda}\left\|\mathbf{j}_{\lambda}\right\|_{2}^{2}+\sum_{\lambda \in \Lambda} \theta_{\lambda}\left(\rho_{\lambda}-v_{\lambda}\right)^{2}\right)
$$

restricted to $v_{\lambda} \geq 0$. Here, $\left(\theta_{\lambda}\right)_{\lambda \in \Lambda},\left(\rho_{\lambda}\right)_{\lambda \in \Lambda}$, and $\left(\omega_{\lambda}\right)_{\lambda \in \Lambda}$ are some suitable positive parameter sequences. The discrepancy $\Delta(\mathbf{j})$ can be obtained by inserting in (10) the current density representation (12) so that

$$
\Delta(\mathbf{j})=\left\|F-T_{\psi} \mathbf{j}\right\|_{2}^{2},
$$

where $F=\left[\left(V_{i}\right)_{i=1, \ldots, N},\left(B_{i}\right)_{i=1, \ldots, M}\right]^{T}$ denotes the measurement vector, while $T_{\psi}$ denotes the matrix whose entries are the coefficients of the operators $\mathcal{V}\left(\mathbf{r}, \mathbf{J}^{p}\right)$ and $\mathcal{B}_{\mathbf{e}}\left(\mathbf{r}, \mathbf{J}^{p}\right)$ w.r.t. $\left(j_{\lambda}^{\ell}\right)_{(\lambda \in \Lambda)(\ell=1,2,3)}$ (see Sec. 4 for details).

The task is to minimize $\mathcal{J}_{\theta, \rho, \omega}(\mathbf{j}, v)$ jointly with respect to both the variables $\mathbf{j}$ and $v$. The first belongs to the space of signals (current densities) to be reconstructed, the second belongs to the space of sparsity indicator weights (see [15, 16] for details).

The minimizer $\left(\mathbf{j}^{*}, v^{*}\right)$ of the functional $\mathcal{J}_{\theta, \rho, \omega}^{(q)}$ subject to the joint sparsity constraints can be approximated by the following iterative algorithm, deduced from [16] (see also [14]). 


\section{Vector Iterative Thresholding Algorithm}

$$
\left\{\begin{array}{l}
\text { Let } \gamma \text { be a suitable relaxation parameter } \\
\text { Choose an arbitrary } \mathbf{j}^{(0)} \in \ell_{2}\left(\boldsymbol{\Lambda} ; \mathbb{R}^{3}\right) \\
\text { For } 0 \leq k \leq K \quad \text { do } \quad \mathbf{j}^{(k+1)}=\mathcal{S}_{\theta, \rho, \omega}^{(q)}\left(\mathbf{j}^{(k)}+\gamma T_{\psi}^{*}\left(F-T_{\psi} \mathbf{j}^{(k)}\right)\right)
\end{array}\right.
$$

The operator $\mathcal{S}_{\theta, \rho, \omega}^{(q)}: \ell_{2}\left(\boldsymbol{\Lambda} ; \mathbb{R}^{3}\right) \rightarrow \ell_{2}\left(\boldsymbol{\Lambda} ; \mathbb{R}^{3}\right)$ is the vector-valued thresholding operator introduced in [15] and can be efficiently evaluated by the algorithm given in [16].

The convergence of the algorithm above can be proved as in [14], nevertheless we expect a slow convergence rate, as already put in evidence in some numerical tests on the solution of a magnetic bidimensional inverse problem [25]. More efficient algorithms can be obtained by choosing an adaptive relaxation parameter. Some strategies to speed-up the convergence rate of Alg. (16) can be found in $[3],[5],[9]$.

\section{Discretization of the forward problem}

In order to implement Alg. (16) we need to solve efficiently the integral equation for $V(\mathbf{r})$ at each iteration step. In neuroscience literature the forward problem is usually solved by a collocation BEM assuming that the primary current $\mathbf{J}^{p}$ can be represented by a sum of current dipoles (see, for instance, $[2,17]$ ). Here, we discretize the boundary integral equations (8) by a Galerkin BEM [21] assuming $\mathbf{J}^{p}$ has the sparse representation (12).

To formulate the Galerkin BEM for the EEG/MEG problem let us introduce the integral operators:

$$
\begin{aligned}
& \left(\mathcal{L}_{B} \mathbf{J}^{p}\right)(\mathbf{r}):=\frac{\mu_{0}}{4 \pi} \int_{G_{0}} \frac{\mathbf{J}^{p}\left(\mathbf{r}^{\prime}\right) \times\left(\mathbf{r}-\mathbf{r}^{\prime}\right)}{\left|\mathbf{r}-\mathbf{r}^{\prime}\right|^{3}} \cdot \mathbf{e}(\mathbf{r}) d \mathbf{r}^{\prime}=-\frac{\mu_{0}}{4 \pi} \int_{G_{0}} \frac{\mathbf{e}(\mathbf{r}) \times\left(\mathbf{r}-\mathbf{r}^{\prime}\right)}{\left|\mathbf{r}-\mathbf{r}^{\prime}\right|^{3}} \cdot \mathbf{J}^{p}\left(\mathbf{r}^{\prime}\right) d \mathbf{r}^{\prime}, \\
& \left(\mathcal{S}_{B} V\right)(\mathbf{r}):=-\frac{\mu_{0}}{4 \pi} \sum_{i=0}^{m}\left(\sigma_{i+1}-\sigma_{i}\right) \int_{S_{i}} V\left(\mathbf{r}^{\prime}\right) \frac{\mathbf{r}-\mathbf{r}^{\prime}}{\left|\mathbf{r}-\mathbf{r}^{\prime}\right|^{3}} \times \mathbf{n}_{i}\left(\mathbf{r}^{\prime}\right) \cdot \boldsymbol{e}(\mathbf{r}) d S_{i}\left(\mathbf{r}^{\prime}\right) \\
& \left(\mathcal{L}_{E} \mathbf{J}^{p}\right)(\mathbf{r}):=\frac{\sigma_{0}}{4 \pi\left(\sigma_{l+1}+\sigma_{l}\right)} \int_{G_{0}} \frac{\mathbf{J}^{p}\left(\mathbf{r}^{\prime}\right) \cdot\left(\mathbf{r}-\mathbf{r}^{\prime}\right)}{\left|\mathbf{r}-\mathbf{r}^{\prime}\right|} d \mathbf{r}^{\prime}, \\
& \left(\mathcal{S}_{E} V\right)(\mathbf{r}):=\frac{1}{4 \pi} \sum_{i=0}^{m} \frac{\left(\sigma_{i+1}-\sigma_{i}\right)}{\left(\sigma_{l+1}+\sigma_{l}\right)} \int_{S_{i}} V\left(\mathbf{r}^{\prime}\right) \frac{\mathbf{r}-\mathbf{r}^{\prime}}{\left|\mathbf{r}-\mathbf{r}^{\prime}\right|^{3}} \cdot \mathbf{n}_{i}\left(\mathbf{r}^{\prime}\right) d S_{i}\left(\mathbf{r}^{\prime}\right) .
\end{aligned}
$$

From (8)-(9) it follows that the unknown function $V(\mathbf{r}) \in \mathcal{H}=L_{2}\left(\cup_{i=0}^{m} \partial G_{i}\right)$ satisfies the Fredholm integral equation of the second kind

$$
\left(\left[\frac{1}{2} I-\mathcal{S}_{E}\right] V\right)(\mathbf{r})=\left(\mathcal{L}_{E} \mathbf{J}^{p}\right)(\mathbf{r}), \quad \mathbf{r} \in \partial G_{l}, \quad l=0, \ldots, m .
$$


The integral operator for the component of the magnetic field along the direction $\mathbf{e}(\mathbf{r})$ can be deduced from (6)-(7),

$$
B(\mathbf{r})=\mathbf{B}(\mathbf{r}) \cdot \mathbf{e}(\mathbf{r})=\left(\mathcal{L}_{B} \mathbf{J}^{p}\right)(\mathbf{r})+\left(\mathcal{S}_{B} V\right)(\mathbf{r}), \quad \mathbf{r} \in \mathbb{R}^{3} \backslash \cup_{i=0}^{m} G_{i} .
$$

The Galerkin BEM consists in finding an element

$$
V_{\mathcal{H}_{\lambda}}(\mathbf{r})=\sum_{\lambda \in \Lambda} v_{\lambda} \psi_{\lambda}(\mathbf{r})
$$

belonging to the space

$$
\mathcal{H}_{\lambda}=\operatorname{span}\left(\left(\psi_{\lambda}\right)_{\lambda \in \Lambda}\right) \subset \mathcal{H}
$$

such that

$$
\sum_{\lambda \in \Lambda} v_{\lambda}\left\langle\left(\frac{1}{2} I-\mathcal{S}_{E}\right) \psi_{\lambda}, \psi_{\mu}\right\rangle=\left\langle\mathcal{L}_{E} \mathbf{J}^{p}, \psi_{\mu}\right\rangle,
$$

for all $\psi_{\mu}, \mu \in \Lambda$. Classical results on boundary element methods (see [21]) allow us to conclude that the Galerkin equations (21) have a unique solution. Moreover, the numerical solution is quasi-optimal, i.e. the following error estimate holds

$$
\left\|V-V_{\mathcal{H}_{\lambda}}\right\|_{\mathcal{H}} \leq c \inf _{V_{\mathcal{H}_{\lambda}} \in \mathcal{H}_{\lambda}}\left\|V-V_{\mathcal{H}_{\lambda}}\right\|_{\mathcal{H}}
$$

The unknown coefficient vector $Y=\left[v_{\lambda}\right]_{\lambda \in \Lambda}$ is the solution to the linear system

$$
A_{E} Y=B_{E},
$$

where

$$
A_{E}=\left[\left\langle\left(\frac{1}{2} I-\mathcal{S}_{E}\right) \psi_{\lambda}, \psi_{\mu}\right\rangle\right]_{\mu, \lambda \in \Lambda},
$$

and

$$
B_{E}=\left[\left\langle\mathcal{L}_{E} \mathbf{J}^{p}, \psi_{\mu}\right\rangle\right]_{\mu \in \Lambda}
$$

For later use it is more convenient to factorize $B_{E}$ as

$$
B_{E}=L_{E} J
$$

where

$$
L_{E}=\left[\left\langle\mathcal{L}_{E} \mathbf{e}^{\ell} \psi_{\lambda}, \psi_{\mu}\right\rangle\right]_{(\mu, \lambda \in \Lambda)(\ell=1,2,3)},
$$

with $\mathbf{e}^{\ell}, \ell=1,2,3$, being the unitary versors of the coordinate system, and

$$
J=\left[j_{\lambda}^{\ell}\right]_{(\lambda \in \Lambda)(\ell=1,2,3)} \cdot
$$

Once the approximation $V_{\mathcal{H}_{\lambda}}$ is evaluated, the magnetic field can be approximated by

$$
B_{\mathcal{H}_{\lambda}}(\mathbf{r})=\left(\mathcal{L}_{B} \mathbf{J}^{p}\right)(\mathbf{r})+\left(\mathcal{S}_{B} V_{\mathcal{H}_{\lambda}}\right)(\mathbf{r})
$$


The entries of the matrix $T_{\psi}$ in Alg. (16) are the coefficients of $B_{\mathcal{H}_{\lambda}}(\mathbf{r})$ and $V_{\mathcal{H}_{\lambda}}(\mathbf{r})$ w.r.t. $\left.\mathbf{j}=\left(j_{\lambda}\right)_{(\lambda \in \Lambda)(\ell=1,2,3}\right)$. To give the explicit expression of $T_{\psi}$, let us write $V_{\mathcal{H}_{\lambda}}$ and $B_{\mathcal{H}_{\lambda}}$ as a function of $\mathbf{j}$. From (20), (23) and (26) it follows that

$$
V_{\mathcal{H}_{\lambda}}(\mathbf{r})=\sum_{\lambda \in \Lambda} \sum_{\mu \in \Lambda} \sum_{\ell=1}^{3}\left(A_{E}^{-1} L_{E}\right)_{\mu \lambda}^{\ell} j_{\mu}^{\ell} \psi_{\lambda}(\mathbf{r}) .
$$

Now, inserting (12) and (30) in (29) we get

$$
B_{\mathcal{H}_{\lambda}}(\mathbf{r})=\sum_{\lambda \in \Lambda} \sum_{\ell=1}^{3}\left(L_{B}(\mathbf{r})\right)_{\lambda}^{\ell} j_{\lambda}^{\ell}+\sum_{\lambda \in \Lambda} \sum_{\mu \in \Lambda} \sum_{\ell=1}^{3}\left(A_{E}^{-1} L_{E}\right)_{\lambda \mu}^{\ell} j_{\mu}^{\ell}\left(\mathcal{S}_{B} \psi_{\lambda}\right)(\mathbf{r}),
$$

where

$$
L_{B}(\mathbf{r})=\left[\left(\mathcal{L}_{B} \mathbf{e}^{\ell} \psi_{\lambda}\right)(\mathbf{r})\right]_{(\lambda \in \Lambda)(\ell=1,2,3)} .
$$

Let

$$
T_{E}=\left[\sum_{\mu \in \Lambda}\left(A_{E}^{-1} L_{E}\right)_{\mu \lambda}^{\ell} \psi_{\mu}\left(\mathbf{p}_{\boldsymbol{i}}\right)\right]_{(i=1, \ldots, N)(\lambda \in \Lambda, \ell=1,2,3)}
$$

and

$$
T_{B}=\left[\left(L_{B}\left(\mathbf{q}_{i}\right)\right)_{\lambda}^{\ell} \psi_{\lambda}\left(\mathbf{q}_{i}\right)+\sum_{\mu \in \Lambda}\left(A_{E}^{-1} L_{E}\right)_{\mu \lambda}^{\ell}\left(\mathcal{S}_{B} \psi_{\mu}\right)\left(\mathbf{q}_{i}\right)\right]_{(i=1, \ldots, M)(\lambda \in \Lambda, \ell=1,2,3)},
$$

by (30) and (31) we obtain

$$
T_{\psi}=\left[\begin{array}{c}
T_{E} \\
T_{B}
\end{array}\right]
$$

We note that Alg. (16) can be implemented efficiently if the matrix $T_{\psi}^{*} T_{\psi}$ can be approximated by a sparse finite matrix. Sparse representations of the integral operators (17) can be obtained by using multiscale bases [6],[7],[24].

\section{$5 \quad$ Numerical Tests}

To give an idea of the behavior of Alg. (16) we consider a simple test problem, i.e. the localization of current dipole sources in a homogeneous spherical conductor $G_{0}$ with radius $R=10 \mathrm{~cm}$. In this case the surface integrals in (29) vanish and the magnetic and electric problems decouple. Nevertheless, the integration of electric and magnetic data allows us to improve the source localization accuracy.

In these tests, the synthetic electromagnetic data are generated by three current dipoles located at a depth of $0.1 R$ below the surface of the sphere. One dipole is radially oriented, so that it does not produce any external magnetic field. The behavior of the electric potential and the radial component of the magnetic field is shown in Fig. 1. To obtain the synthetic measurements, the 
radial component of the magnetic field has been sampled on $M=400$ sites, distributed on a sphere of radius $1.1 R$ concentric to $G_{0}$, while the electric potential has been sampled on $N=100$ sites, located on the sphere surface. The electric and magnetic data are scaled in order to have the same norm. Finally, a white noise with linear snr equal to 1 has been added. The synthetic noisy data and the sensor distribution are shown in Fig. 2.

The spherical conductor has been parametrized in a spherical coordinate system and a linear finite element space with $64^{3}$ degrees of freedom has been used as approximation space. The inverse problem has been solved by the iterative thresholding algorithm (16) with 20 iteration steps. Finally, the acceleration strategy proposed in [3] has been used to speed-up the convergence rate.

In Fig. 3 (left) the intensity of the current density reconstructed by Algorithm (16) is displayed. For comparison, the results obtained by classical Tikhonov regularization are also shown (Fig. 3, right). A qualitative analysis of the figures show that the proposed algorithm allows us to better focus the current sources, while Tikhonov regularization produces a more blurred image with fictitious sources.

In Tab. 1 the localization error (LE) and the spatial dispersion (SD) are shown for each dipole source. Let $\sigma$ be the source location and $I(\mathbf{r})$ be the intensity of the reconstructed current density. The LE is the distance between $\sigma$ and the location of the maximum of $I(\mathbf{r})$ in the neighborhood of the source; the SD is evaluated as $\|d(\mathbf{r}) I(\mathbf{r})\|_{2} /\|I(\mathbf{r})\|_{2}$. Smaller the values of LDE and SD, the higher accuracy and smaller spread.

\begin{tabular}{|l|c|c|}
\hline & Alg. (16) & $\begin{array}{c}\text { Tikhonov } \\
\text { method }\end{array}$ \\
\hline LDE (first source) & $2.5 \mathrm{~mm}$ & $3.4 \mathrm{~mm}$ \\
\hline SD (first source) & $0.06 \mathrm{~mm}$ & $0.48 \mathrm{~mm}$ \\
\hline \hline LDE (second source) & $1.9 \mathrm{~mm}$ & $3.5 \mathrm{~mm}$ \\
\hline SD (second source) & $0.15 \mathrm{~mm}$ & $0.22 \mathrm{~mm}$ \\
\hline \hline LDE (third source) & $8.0 \mathrm{~mm}$ & $9.7 \mathrm{~mm}$ \\
\hline SD (third source) & $0.18 \mathrm{~mm}$ & $0.31 \mathrm{~mm}$ \\
\hline
\end{tabular}

Table 1. Localization error (LE) and spatial dispersion (SD).

It is interesting to observe that magnetic data or electric data give rise to inaccurate localization when used uncoupled (see Fig. 4).

Even if this preliminary experiment has a very simple geometry and uses a single approximation scale, the results show that both integration of electric and magnetic data and joint sparsity allow to improve localization accuracy. Next step will be to implement the algorithm on a real head geometry and to solve the EEG/MEG problem using real high-level noisy data. When we deal with real-life applications, we face some difficulties that require more sophisticated numerical techniques. First of all spherical coordinate system may present sin- 
gularities, so that we need more efficient parametrization of the head. Moreover, it would be useful to dispose of multiscale bases especially designed for representing neuroelectric currents. Finally, the processing of high noise electro- and magneto-encephalographic signals require suitable estimator.
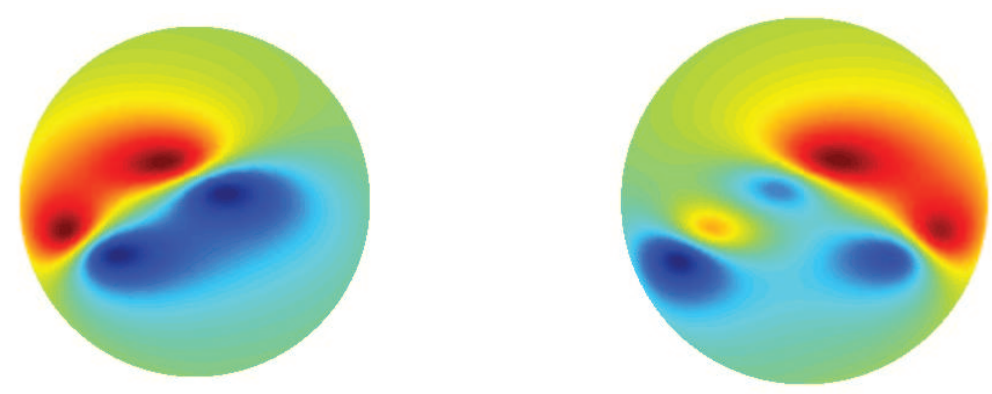

Fig. 1. Synthetic electric potential (left) and magnetic field (right).
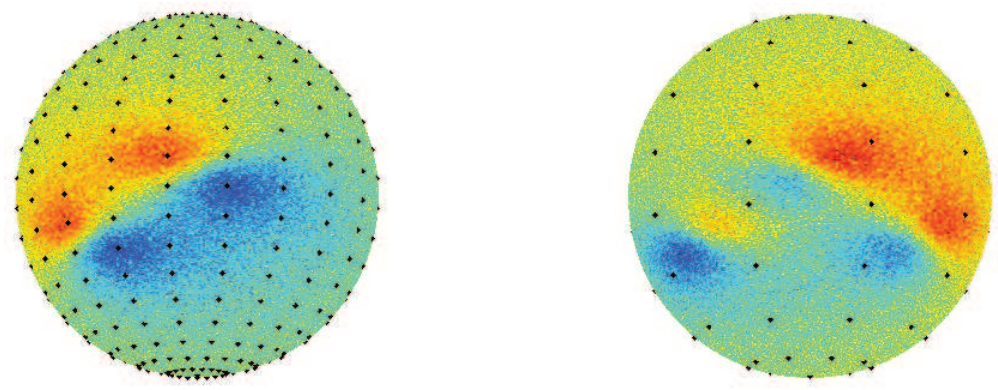

Fig. 2. Synthetic noisy electric potential (left) and magnetic field (right). The sensor sites are displayed as black points.

\section{References}

1. Aldoubi, A., Unser, M., Laine, A. (eds.): Wavelets in Biomedical Imaging. IEEE Trans. on Medical Imaging 22, 285-288 (2003)

2. Babiloni, F., Carducci, F., Cincotti, F., Del Gratta, C., Pizzella, V., Romani, G.L., Rossini, P.M., Tecchio, F., Babiloni, C.: Linear Inverse Source Estimate of Combined EEG and MEG Data Related to Voluntary Movements. Hum. Brain Mapp. 14, 197-209 (2001)

3. Beck, A., Teboulle, M.: A fast iterative shrinkage-thresholding algorithm for linear inverse problems. SIAM J. Imaging Sci. 2, 183-202 (2009) 

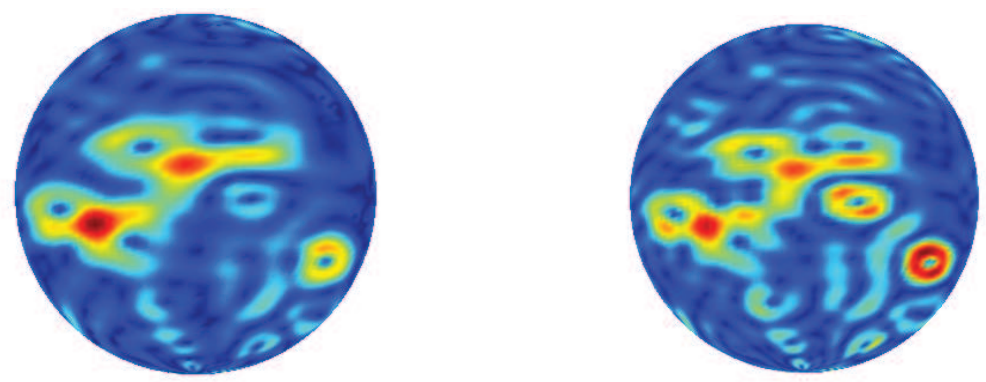

Fig. 3. The intensity of the reconstructed current density obtained by Alg. (16) (left) and by Tikhonov regolarization (right) when using the noisy data.
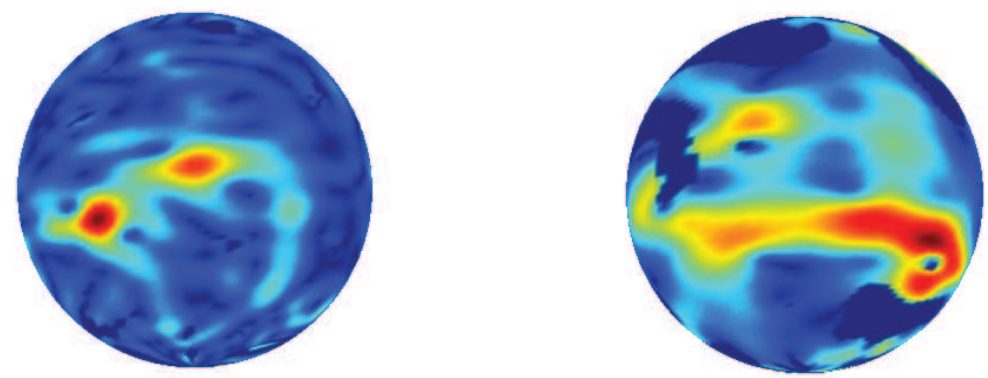

Fig. 4. The intensity of the reconstructed current density obtained by using just magnetic (left) or electric (right) noisy data in Alg. (16). 
4. Bertero, M., Boccacci, P., Introduction to Inverse Problems in Imaging. Institute of Physics, Bristol, Philadelphia (2002)

5. Bretti G., Fornasier, M., Pitolli, F.: Electric current density imaging via an accelerated iterative algorithm with joint sparsity constraints. In Proc. SPARS09 (St-Malo, France), http://hal.inria.fr/SPARS09/en

6. Cohen A., Hoffmann, M., Reiss, M.: Adaptive wavelet Galerkin methods for linear inverse problems, SIAM J. Numer. Anal. 42, 1479-1501 (2004)

7. Dahmen, W., Harbrecht, H., Schneider, R.: Adaptive methods for boundary integral equations: complexity and convergence estimates. Math. Comp. 76, 1243-1274 (2007)

8. Daubechies, I., Defrise, M., De Mol, C.: An Iterative Thresholding Algorithm for Linear Inverse Problems with a Sparsity Constraint. Commun. Pure Appl. Math $57,1413-1457$ (2004)

9. Daubechies, I., Fornasier, M., Loris, I.: Accelerated Projected Gradient Method for Linear Inverse Problems with Sparsity Constraints. J. Fourier Anal. Appl. 14, 764-792 (2008)

10. Del Gratta, C., Pizzella, V., Tecchio, F., Romani, G.L.: Magnetoencephalography - A Noninvasive Brain Imaging Method with 1ms Time Resolution. Rep. Prog. Phys. 64, 1759-1814 (2001)

11. Donoho, D.L.: Superresolution via Sparsity Constraints. SIAM J. Math. Anal. 23, 1309-1331 (1992)

12. Donoho, D.L.: De-noising by Soft-Thresholding. IEEE Trans. Inform. Theory 41, 613-627 (1995)

13. Engl, H.W., Hanke, M., Neubauer, A., Regularization of Inverse Problems. Kluwer, Dordrecht (2000)

14. Fornasier, M., Pitolli, F.: Adaptive Iterative Thresholding Algorithms for Magnetoencephalography (MEG). J. Comput. Appl. Math. 221, 386-395 (2008)

15. Fornasier, M., Rauhut, H.: Iterative Thresholding Algorithms. Appl. Comput. Harmon. Anal. 25, 187-208 (2008)

16. Fornasier, M., Rauhut, H.: Recovery Algorithms for Vector Valued Data with Joint Sparsity Constraints. SIAM J. Numer. Anal. 46, 577-613 (2008)

17. Fuchs, M., Wagner, M., Kastner, J.: Boundary Element Method Volume Conductor Models for EEG Source Reconstruction. Clin. Neurophysiol. 112, 1400-1407 (2001)

18. Geselowitz, D.B.: On bioelectric potentials in an inhomogeneous volume conductor. Biophys. J. 7, 1-11 (1967)

19. Geselowitz, D.B.: On the magnetic field generated outside an inhomogeneous volume conductor by internal current sources. IEEE Trans. Magn. 6, 346347 (1970)

20. Hamalainen, M.S., Hari, R., Ilmoniemi, R.J., Knuuttila, J., Lounasmaa, O.V.: Magnetoencephalography - Theory, Instrumentation and Applications to Non Invasive Studies of the Working Buman brain. Rev. Mod. Phys. 65, 413-497 (1993)

21. Hsiao, G.C., Wendland, W.L., Boundary Element Methods: Foundation and Error Analysis. In: Stein, E., de Borst, R., Hughes, T.J.R. (eds.), Encyclopedia of Computational Mechanics, pp. 339-373. John Wiley \& Sons, New York (2004)

22. Kaipio, J., Somersalo E.: Statistical and computational inverse problems. Applied Mathematical Sciences 160, Springer, New York (2005)

23. Mallat, S.: A Wavelet Tour on Signal Processing. The Sparse Way. Third edition. Elsevier/Academic Press, Amsterdam (2009)

24. von Petersdorff, T., Schwab, C., Schneider, R.: Multiwavelets for second-kind integral equations. SIAM J. Numer. Anal. 34, 2212-2227 (1997) 
25. Pitolli, F., Bretti, G.: An Iterative Algorithm with Joint Sparsity Constraints for Magnetic Tomography. In: Daehlen, M., et al. (eds.), Mathematical Methods for Curves and Surfaces. Lecture Notes in Computer Science 5862, pp. 316-328. Springer-Verlag (2010)

26. Sarvas, J.: Basic Mathematical and Electromagnetic Concepts of the Biomagnetic Inverse Problem. Phys. Med. Biol. 32, 11-22 (1987) 